\title{
Molecular analysis of circulating tumor DNA from breast cancer patients before and after surgery and following adjuvant chemotherapy
}

\author{
KATARINA ZELINOVA $^{1,2}$, MARIANNA JAGELKOVA ${ }^{1,2}$, ZUZANA LAUCEKOVA $^{2}$, \\ MARTINA BOBROVSKA ${ }^{3}$, ZUZANA DANKOVA $^{1}$, MARIAN GRENDAR ${ }^{4}$ and KAROL DOKUS ${ }^{2,5}$ \\ ${ }^{1}$ Division of Oncology, Biomedical Center Martin; Departments of ${ }^{2}$ Obstetrics and Gynecology and \\ ${ }^{3}$ Pathological Anatomy, Jessenius Faculty of Medicine in Martin, Comenius University in Bratislava, Martin University Hospital; \\ ${ }^{4}$ Department of Bioinformatics, Biomedical Center Martin, Jessenius Faculty of Medicine in Martin, Comenius University \\ in Bratislava, SK-036 01 Martin; ${ }^{5}$ Second Department of Obstetrics and Gynecology, Slovak Medical University, \\ Faculty Hospital with Polyclinic of F.D. Roosevelt, 97517 Banska Bystrica, Slovakia
}

Received August 1, 2019; Accepted June 11, 2020

DOI: $10.3892 / \mathrm{mco} .2020 .2096$

\begin{abstract}
The primary aim of the present study is to provide a complex molecular profile of tumors using liquid biopsy and to monitor profile changes over time in association with surgery and administered adjuvant therapy. Our secondary aim was to compare the liquid biopsy profile with the tissue biopsy and assess concordance. A total of 27 samples of circulating tumor DNA (ctDNA) collected from 9 breast cancer patients at three different time points and their matched formalin-fixed and paraffin-embedded (FFPE) samples of primary tumor were analyzed with targeted next-generation sequencing. Somatic pathogenic variants were detected before surgery in samples from 5 patients $(55.6 \%)$. The most frequently mutated genes were phosphatase and tensin homolog $(4 / 9,44.4 \%)$ and tumor protein $53(4 / 9,44.4 \%)$. Serial sampling of ctDNA enabled the detection of more variants compared with single-time tissue primary tumor biopsy. There were 17 ctDNA variants across all samples, but only 6 FFPE variants across all patients. In addition, the concordance between ctDNA and FFPE DNA was determined in only 1 patient, and this was connected with higher variant allele frequency. The findings of the present study suggest that liquid biopsy and tissue biopsy may be used as complementary analyses to adequately capture all tumor variants.
\end{abstract}

Correspondence to: Dr Katarina Zelinova, Division of Oncology, Biomedical Center Martin, Jessenius Faculty of Medicine in Martin, Comenius University in Bratislava, 4C Mala Hora Street, SK-036 01 Martin, Slovakia

E-mail: katarina.zelinova@uniba.sk

Key words: liquid biopsy, circulating tumor DNA, breast cancer, serial monitoring, next-generation sequencing

\section{Introduction}

Breast cancer $(\mathrm{BC})$ is the most commonly diagnosed carcinoma in women, accounting for almost 1 in 4 female cancers, and it is the leading cause of cancer-related mortality in women globally (1). BC is a complex heterogenous disease defined by its diverse aggressive behavior, potential to metastasize and therapeutic gene targets. The primary tumor generally contains combined diverse clonal populations of cells; therefore, invasive biopsy of the solid tumor may be biased and provide only an incomplete molecular profile of the analyzed tumor (2).

Circulating tumor DNA (ctDNA) has the potential to capture complete information on genetic alterations, including the somatic single-nucleotide variations present in the tumor and its metastases, and also to longitudinally monitor these mutations throughout the course of patient treatment $(3,4)$. In addition, ctDNA is considered to be released from apoptotic and necrotic tumor cells, and its blood circulation half-life ranges from minutes to several hours, which makes it a dynamic, almost real-time, biomarker for cancer monitoring $(5,6)$. The detection of ctDNA, however, presents a serious challenge, as ctDNA is highly fragmented to on average $170 \mathrm{bp}$ in length $(7,8)$. ctDNA may occasionally be present only as a minimal fraction in the circulation compared with the wild-type cell-free DNA (cfDNA) released from normal cells (4). In healthy individuals, cfDNA originates from hematopoietic cells $(9,10)$. However, ctDNA of cancer patients is tissue-specific, as the majority of their ctDNA comes from the tumor tissue of origin (9-11). The ratio of ctDNA to cfDNA depends on tumor and immunological factors, tumor burden and progression, and blood clearance mechanisms $(12,13)$.

Targeted next-generation sequencing (NGS), which generates sufficiently high coverage over a certain region, is therefore required, and ctDNA molecular analysis and identification of these low-frequency variants is important for detection of cancer in its early stages and appropriate diagnosis. Moreover, ctDNA analysis in the clinical setting may 
guide decision-making and potentially facilitate personalized cancer care $(8,14,15)$.

In $\mathrm{BC}$, recent studies have indicated the versatile role of ctDNA analysis in determining the genomic profile of tumors (16-19), to sensitively and specifically identify preclinical metastases and predict a relapse ahead of its diagnosis (20-22), and to asses resistance to targeted therapy or to uncover tumor heterogeneity (23).

The primary aim of the present study was to provide a complex tumor molecular profile with liquid biopsy and to monitor its changes over time in association with surgery and adjuvant therapy. The secondary aim was to compare this liquid biopsy profile with the tissue biopsy and to assess concordance.

\section{Materials and methods}

Patients. Patients with invasive BC were treated at the Clinic of Gynecology and Obstetrics at the Jessenius Faculty of Medicine in Martin, Comenius University in Bratislava (JFM CU) and University Hospital in Martin (UHM). All subjects provided their written informed consent and the study was conducted in accordance with the Declaration of Helsinki and following the protocol approved by the Ethics Committee of JFM CU (IORG0004721) under project identification code EC 1525/2014. The patient data were collected retrospectively from medical records.

Blood sample processing. Serial peripheral whole-blood samples were drawn from 9 patients at three different timepoints: Before surgery, 2 days after surgery and again 7 months following adjuvant chemotherapy. Briefly, $10 \mathrm{ml}$ blood samples $(n=27)$ were collected in $\mathrm{K}_{3}$ EDTA tubes (Sarstedt AG \& Co.) and processed within $2 \mathrm{~h}$. Plasma samples were separated by two-step centrifugation at 2,200 $\mathrm{x} \mathrm{g}$ for $8 \mathrm{~min}$ at $4^{\circ} \mathrm{C}$ and $20,000 \mathrm{x} \mathrm{g}$ for $8 \mathrm{~min}$ at $4^{\circ} \mathrm{C}$. The plasma samples were then stored at $-80^{\circ} \mathrm{C}$ until DNA extraction.

DNA extraction and quantification . ctDNA was isolated from $3 \mathrm{ml}$ plasma by the commercially available QIAamp DSP Virus kit (Qiagen $\mathrm{GmbH}$ ), according to the manufacturer's instructions, and with the QIAvac 24 Plus vacuum manifold (Qiagen $\mathrm{GmbH})$. The plasma samples were lysed, ctDNA was bound to a silica-based membrane, washed with ethanol-containing buffers, and waste was drained by vacuum pressure. The ctDNA was eluted in $30 \mu \mathrm{l}$ sterile distilled water and ctDNA concentration was quantified with the Qubit dsDNA HS Assay kit (Invitrogen; Thermo Fisher Scientific, Inc.) on the Qubit 2.0 Fluorometer (Invitrogen; Thermo Fisher Scientific, Inc.). The extracted ctDNA was then concentrated using the CentriVap Centrifugal concentrator 78100 (Labconco) at $37^{\circ} \mathrm{C}$ for $90 \mathrm{~min}$ and ctDNA was re-suspended in $30 \mu 1$ sterile distilled water. Finally, the resultant ctDNA samples were stored at $-20^{\circ} \mathrm{C}$ until analysis.

DNA was also isolated from 9 formalin-fixed and paraffin-embedded (FFPE) tissue samples obtained from the Department of Pathological Anatomy of JFM CU and UHM. The commercially available BlackPREP FFPE DNA kit (Analytic Jena AG) was used according to the manufacturer's instructions. In brief, FFPE slides were lysed and shaken at $65^{\circ} \mathrm{C}$ for $1 \mathrm{~h}$, followed by $1 \mathrm{~h}$ at $90^{\circ} \mathrm{C}$. DNA was bound to a spin filter membrane and washed with ethanol-based buffers and then eluted in $50 \mu \mathrm{l}$ elution buffer. The DNA concentration was quantified with the Qubit dsDNA BR Assay kit (Invitrogen; Thermo Fisher Scientific, Inc.) on the Qubit 2.0 Fluorometer (Invitrogen; Thermo Fisher Scientific, Inc.SA) and DNA samples were stored at $-20^{\circ} \mathrm{C}$ until analysis.

DNA library preparation. DNA libraries $(n=35)$ for NGS were prepared using the TruSight Tumor 26 kit (Illumina, Inc.) according to the manufacturer's instructions. Briefly, the quality and the amplification potential of DNA extracted from FFPE samples were determined by quantitative (q)PCR analysis using the KAPA SYBR FAST qPCR Universal Master Mix kit (Kapa Biosystems; Roche Diagnostics). A $\Delta \mathrm{Cq}$ value was calculated for each sample by comparing the amplification potential of FFPE DNA with the amplification potential of reference QCT DNA provided in the kit. DNA libraries $(\mathrm{n}=8)$ were prepared with either $20 \mu \mathrm{l}$ of neat FFPE DNA or $20 \mu$ l diluted FFPE DNA depending on the $\Delta \mathrm{Cq}$ value of the corresponding sample (Table I). Although sample 7.2 had low concentration, the DNA library could be prepared; however, sample 9.2 had almost no DNA detected on Qubit assay and was therefore excluded from further analysis. The ctDNA sample quality was not investigated, and the DNA libraries $(\mathrm{n}=27)$ were prepared with $20 \mu 1$ neat ctDNA.

FPA and FPB, two separate complementary oligonucleotide pools, were hybridized to DNA samples overnight. This process resulted in generation of $\mathrm{A}$ and $\mathrm{B}$ complementary DNA libraries for every DNA sample by targeting the positive and the negative DNA strands of the same region. The oligonucleotide pools are specific to the targeted regions of interest in 174 amplicons of 26 genes (Table II). Unbound oligonucleotides were removed, and bound oligonucleotides were extended and ligated. PCR amplification with indices and adapters added to extension-ligation products was then performed, and the libraries were purified using AMPure XP magnetic beads (Beckman Coulter, Inc.). The libraries were quantified using Agilent High Sensitivity DNA Kit (Agilent Technologies, Inc.) on the 2100 Bioanalyzer (Agilent Technologies, Inc.) according to the manufacturer's instructions, and DNA library concentrations were then quantified by the Qubit dsDNA HS Assay kit (Invitrogen; Thermo Fisher Scientific, Inc.) on the Qubit 2.0 Fluorometer (Invitrogen; Thermo Fisher Scientific, Inc.).

DNA sequencing and data analysis. Targeted amplicon sequencing was performed on MiSeq (Illumina, Inc.), with MiSeq Control Software version 2.6.2, according to the manufacturer's instructions. Only four library A and four library B samples could be sequenced per run. In brief, the libraries were normalized to $4 \mathrm{nM}$ and pooled into a single tube. Then, $600 \mu \mathrm{l}$ of pooled libraries were loaded into a MiSeq reagent cartridge v2 (300 cycles) and sequenced on flow cell using $150 \mathrm{bp}$ paired-end sequencing protocol. The raw sequence data were de-multiplexed and converted into FASTQ files using MiSeq Reporter version 2.6. The sequencing reads were aligned to the Genome Reference Consortium Human Build 37 (GRCh37)/Human Genome version 19 (hg19). Variants were called by Somatic Variant Caller Version 3.2.1 and written 
Table I. $\Delta \mathrm{Cq}$ values of formalin-fixed and paraffin-embedded DNA samples with the corresponding dilution.

\begin{tabular}{lcc}
\hline Sample & $\Delta \mathrm{Cq}$ & Dilution \\
\hline 1.2 & 0.98 & $2 \mathrm{x}$ \\
2.2 & 3.63 & No dilution \\
3.2 & 3.42 & No dilution \\
4.2 & -0.16 & $4 \mathrm{x}$ \\
5.2 & 2.17 & No dilution \\
6.2 & 2.45 & No dilution \\
7.2 & Low DNA concentration & No dilution \\
8.2 & 0.17 & $4 \mathrm{x}$ \\
9.2 & No DNA & \\
\hline
\end{tabular}

Table II. Genes included in TruSight Tumor 26 kit (Illumina, Inc.).

\begin{tabular}{llll}
\hline AKT1 & ERBB2 & KRAS & PTEN \\
ALK & FBXW7 & MAP2K1 & SMAD4 \\
APC & FGFR2 & MET & SRC \\
BRAF & FOXL2 & MSH6 & STK11 \\
CDH1 & GNAQ & NRAS & TP53 \\
CTNNB1 & GNAS & PDGFRA & \\
EGFR & KIT & PIK3CA & \\
\hline
\end{tabular}

into VCF files. Variants with variant allele frequency (VAF) $<3.0 \%$, quality score $<30$, low depth of coverage or significant strand bias were filtered out, and strand bias filler removed sequencing artifacts that were asymmetrically represented only in one strand. The resulting variants were annotated using the open source Variant Effect Predictor version 93 bioinformatics software tool (24). The classification of variants was based on VarSome v6.7, which uses the American College of Medical Genetics and Genomics guidelines $(25,26)$. The yielded pathogenic variants were subsequently checked against the Catalogue of Somatic Mutations in Cancer (COSMIC) database version 88 for somatic categorization (27). Finally, variants of unknown significance or germline and benign variants were filtered out.

\section{Results}

Patient characteristics. A total of 9 female patients with histopathologically confirmed $\mathrm{BC}$ were analyzed. The mean age was $56.22 \pm 9.62$ years and the histopathological characteristics of the patients are summarized in Table III. A complete list of the patients' characteristics is provided in Table SI. Most patients $(55.6 \%)$ had invasive ductal carcinoma (IDC) and all tested samples were estrogen receptor (ER)-positive. Only 2 samples were human epidermal growth factor receptor 2 (HER-2)-positive and the HER-2 status of 2 patients was unavailable. The molecular subtypes were luminal A (44.4\%) or luminal B (44.4\%), and the molecular subtype of 1 patient was unknown due to missing data.
Table III. Histopathological characteristics of patients with BC.

\begin{tabular}{ll}
\hline Characteristics & $\mathrm{N}(\%)$ \\
\hline Histological type & \\
IDC & $5(55.6)$ \\
ILC & $4(44.4)$ \\
Histological grade & \\
G1 & $2(22.2)$ \\
G2 & $4(44.4)$ \\
G3 & $3(33.3)$ \\
ER status & \\
Positive & $9(100.0)$ \\
Negative & $0(0.0)$ \\
PR status & \\
Positive & $8(88.9)$ \\
Negative & $1(11.1)$ \\
HER-2 status & \\
Positive & $2(22.2)$ \\
Negative & $5(55.6)$ \\
Unknown & $2(22.2)$ \\
Ki-67 status & \\
Low & $5(55.6)$ \\
High & $4(44.4)$ \\
Molecular subtype & \\
Luminal A & $4(44.4)$ \\
Luminal B & $4(44.4)$ \\
Unknown & $1(11.1)$ \\
\hline
\end{tabular}

$\mathrm{BC}$, breast cancer; IDC, invasive ductal carcinoma; ILC, invasive lobular carcinoma; ER, estrogen receptor; PR, progesterone receptor; HER-2, human epidermal growth factor receptor 2; Ki-67 proliferation index.

Molecular analysis of ctDNA and FFPE DNA. We monitored somatic variants with pathogenic effect in ctDNA from three different time points and FFPE samples in 9 patients with BC across 26 genes (Table IV). Each patient had at least one somatic pathogenic variant. The majority of somatic pathogenic variants were found in samples prior to surgery in 5 patients $(55.6 \%)$. No somatic pathogenic variants were detected in the patients' follow-up samples, postoperatively or after adjuvant therapy. The patients had low-grade (G1 or G2) $\mathrm{BC}$, and 4 had luminal A subtype.

By contrast, somatic pathogenic variants were detected only in postoperative samples in patients 4 and 5 (22.2\%). The patients had higher-grade (G2 or G3) cancer, unknown HER-2 status and luminal B or unknown luminal type. Patients 2 and 6 had somatic pathogenic variants only in samples after chemotherapy (22.2\%). These patients had luminal B, HER-2 ${ }^{+}$and grade $3 \mathrm{BC}$.

No somatic pathogenic variants were detected in FFPE DNA samples of patients 3 and 6. Complete accord between ctDNA and FFPE DNA was observed only in patient 4 (11.1\%). 


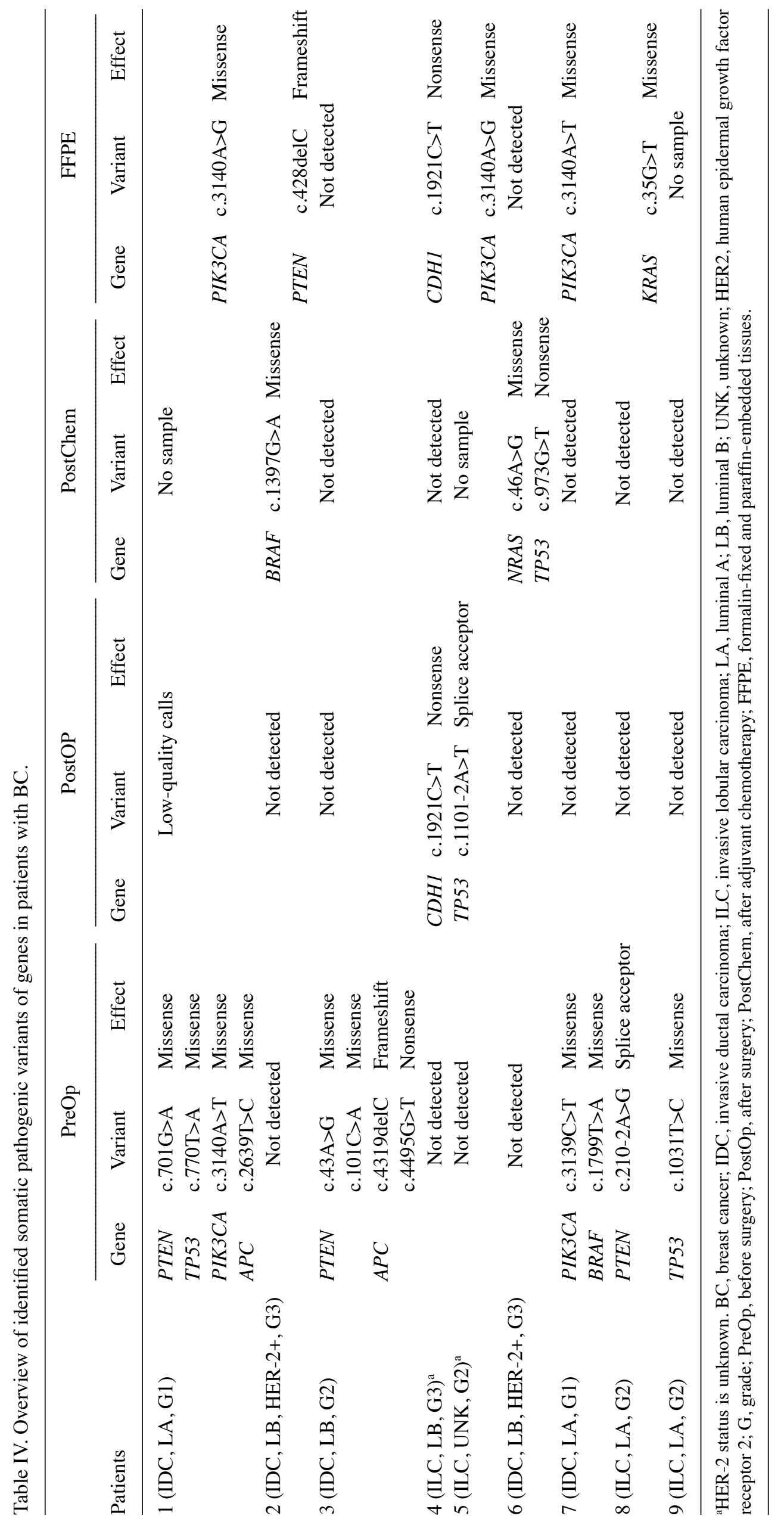


Although phosphatidylinositol-4,5-bisphosphate 3-kinase catalytic subunit alpha (PIK3CA) mutations in codon 1,047 were found in patient 1 and 7 ctDNA and FFPE DNA, the protein variants were different: p.His1047Leu in the ctDNA and p.His1047Arg in FFPE in patient 1, and p.His1047Tyr in the ctDNA and p.His1047Leu in FFPE in patient 7. In addition, while patient 6 displayed negative concordance with no somatic pathogenic variants detected in the ctDNA sample before surgery or the FFPE sample, two somatic pathogenic alterations were detected in the ctDNA sample after chemotherapy. Patient 9 FFPE sample was excluded from DNA library preparation due to insufficient DNA concentration. Two ctDNA libraries, post-chemotherapy ctDNA samples of patient 1 and 5, were not sequenced because of technical difficulties. Finally, the postoperative ctDNA sample of patient 1 generated only low-quality calls, which were filtered out.

Overall, 23 variants in 8 different genes were detected in the present study, and these were mostly missense mutations $(15 / 23,65.22 \%)$. The most common mutation type was single nucleotide substitution $(21 / 23,91.3 \%)$, and the most frequently mutated genes were phosphatase and tensin homolog (PTEN; 4/9, 44.4\%), tumor protein 35 (TP53; 4/9, 44.4\%) and PIK3CA (3/9, 33.3\%; Table SII).

The mean depth of coverage per targeted region in the ctDNA samples was $12,926 \pm 8,120$ (range, 2,261-29,762). The mean variant allele frequency (VAF) in ctDNA samples was $4.74 \pm 1.95 \%$ (range, 3.04-10.43\%) (Table SIII). The mean depth of coverage per targeted region in FFPE DNA samples was $25,169 \pm 9,533$ (range, 12,881-40,713). Finally, the mean VAF in FFPE DNA samples was $25.91 \pm 15.15 \%$ (range, $8.04-50.53 \%$; Table SIV).

\section{Discussion}

In the present study, the molecular profile in serial ctDNA samples from BC patients was monitored and it was demonstrated that ctDNA sequencing provides dynamic information on somatic variants with pathogenic effects in tumors. The molecular analysis revealed that patients with lower-grade and luminal A BC subtype and 1 patient with low-grade luminal B subtype had somatic pathogenic variants detected only in their ctDNA prior to surgery. This correlates with a generally better prognosis of the luminal A subtype compared with other BC subtypes (28-30). Detection of somatic pathogenic variants in ctDNA only after surgery was associated with patients of unknown HER-2 status and luminal B, and unknown luminal type; but these patients had higher-grade (G2 or G3) cancer. While patients with luminal B, HER $-2^{+}$and grade 3 had somatic pathogenic variants detected in ctDNA only after chemotherapy, restricted patient numbers, lack of characterization and impossibility of analyzing certain samples prevented significant conclusions from these results.

The number of our patients was restricted as only a limited number of BC patients admitted to the hospital was able to comply with the demanding serial sampling.

Another limitation of the present study was the exclusion of important BC genes, such as BRCA1, BRCA2 and ESRI. We selected TruSight Tumor 26 kit with the panel of 26 oncogenes and tumor suppressor genes, which are the most frequently altered in solid cancers. This kit was designed to detect somatic variants in highly degraded and fragmented samples, such as FFPE slides, and it was inferred that it would also be suitable for fragmented ctDNA samples.

Although detecting somatic variants without matched normal tissue sample is possible, albeit difficult, VAF denoting the number of reads that support the mutated allele at a given locus can help distinguish somatic from germline variants in both tumor samples and ctDNA. In theory, the somatic variants should only be present in the tumor cells and, thus, give a low VAF in ctDNA, whereas the germline variants would be present in both the sample tumor and normal cells, resulting in a VAF of $\sim 50 \%$ for heterozygous variants and $\sim 100 \%$ for homozygous (31). In addition, the VAF of somatic variants in analyzed tissues depends on the numbers of tumor and normal cells, and this enables their possible detection at any level (32).

The variants herein were considered somatic if they were included in version 88 of the COSMIC database (27). While we were able to detect variants with low VAF in the ctDNA and FFPE samples, concordance between ctDNA and FFPE DNA was only determined in 1 patient. This patient had a higher VAF compared with other patients' detected variants, and the variant detected in the $C D H 1$ gene had both the highest VAF in ctDNA (10.43\%) and FFPE DNA (50.53\%). Consistently with these findings, Chae et al also recorded this connection between higher VAF and concordant mutations in ctDNA and tissue biopsy from a patient with BC (17).

Somatic PTEN mutations in $\mathrm{BC}$ are rare, with a reported frequency of 5-10\% (33-35). The frequency of PTEN alterations in our patients was $44.4 \%$. It is hypothesized that the difference may be caused by restricted number of our patients. There was no association between PTEN alterations and distinctive clinicopathological characteristics in our patients. However, there is no consistent evidence to prove the real prognostic role of PTEN mutations in BC due to the lack of reproducibility in studies (35). The frequency of TP53 mutations in BC is $\sim 40 \%(33,36)$. TP53 mutations were detected in $44.4 \%$ of our patients. There was no association between TP53 mutations and distinctive clinicopathological characteristics in our patients. However, several studies demonstrated that TP53 mutations occur more frequently in HER-2-enriched tumors compared with luminal A or luminal B tumors $(37,38)$. By contrast, high frequency of PIK3CA mutations (33.3\%) in our patients was correlated with $30-40 \%$ prevalence of PIK3CA mutations in patients with BC $(33,39)$. Our patients with PIK3CA had lower-grade tumors. The association of PIK3CA mutations and lower-grade $\mathrm{ER}^{+} \mathrm{BC}$ was shown in the analysis of pooled data by Zardavas et al (40).

Alborelli et al (41), analyzed cfDNA of 114 clinically healthy individuals for genetic alterations with a limit of detection as low as $0.08 \%$ of VAF. The majority of the subjects (84\%) had no genetic alterations in cfDNA. However, the authors identified pathogenic cancer alterations in 4 healthy individuals. The alterations were detected in cancer hotspot variants, including PIK3CA p.His1047Arg. These individuals developed a benign neoplasm or invasive breast in the 10 years following blood collection. Therefore, the analysis of cfDNA in healthy subjects may be relevant to the early detection of cancer.

Serial sampling of ctDNA enabled detection of more variants than in one-time primary tumor tissue biopsy. The results determined 17 ctDNA variants in all samples, but 
only 6 FFPE variants in all patients. The different number of detected variants and their diversity may be attributed to intratumor heterogeneity, where ctDNA may be released into the bloodstream from a completely unique cell subpopulation with mutations different to those present in the primary tumor (2). Moreover, alterations identified only in ctDNA may be missed by single-site tissue biopsy due to sampling different tumor areas and incomplete genetic profiling (42). Mutations with lower VAF identified only in FFPE samples may be unaccounted for in ctDNA analysis due to their under-representation in the circulation. Therefore, we suggest that liquid biopsy and tissue biopsy should be complementary analyses with combined capability of capturing all tumor variants.

The advantages of ctDNA analysis and the liquid biopsy were also demonstrated by Finzel et al (43), who studied data of 351 patients with stage IV solid tumors, including BC, focusing on the discordant gene mutations identified between FFPE blocks and ctDNA from blood samples. The authors detected different molecular alterations between tissue and liquid biopsies in $86 \%$ of patients. However, $42 \%$ of the mutations detected only in the liquid biopsy had clinical relevance in prediction of sensitivity or resistance to administered targeted therapies. The combined assessment of liquid and solid biopsies enables better characterization of tumor heterogeneity and important information for patient therapy (43).

Rodriguez et al analyzed PIK3CA and TP53 mutations in matched ctDNA samples before biopsy and tumor biopsy samples in patients with early BC. Concordance between ctDNA mutations and biopsy mutations was observed in only $8 / 29$ (27.6\%) patients. All patients with concordant mutations had higher-grade disease. Somatic PIK3CA mutations were identified in 19/29 (65.5\%) patients. TP53 mutations were identified in 6/29 (20.7\%), and 4 patients (13.8) had both PIK3CA and TP53 mutations. Moreover, the authors identified 4 additional mutations in ctDNA, which were not detected in tissue biopsy. They proposed that ctDNA analysis could identify tumor heterogeneity, improve the diagnosis of early BC patients, and provide significant information (44).

Similar studies have been performed in $\mathrm{ER}^{+}$and triple-negative $\mathrm{BC}$, but these studies used different methods, or mainly focused on comparing tissue biopsy results with the ctDNA detected in metastatic disease $(16,45,46)$. In a prospective study, Beaver et al (16) analyzed 30 primary breast tumor samples for frequently occurring PIK3CA mutations. Therein, they used Sanger sequencing and matched pre- and postoperative plasma tumor DNA (ptDNA) from 29 patients with early-stage BC by droplet digital PCR (ddPCR) analysis. This ddPCR analysis verified all the mutations detected in the tumor by Sanger sequencing and identified five new mutations, with concordance between tumor and preoperative ptDNA observed in 14/15 PIK3CA mutations. A total of 10 patients were mutation-positive in preoperative ptDNA samples, and 5 of them were also mutation-positive in postoperative ptDNA. This study demonstrated precise mutation detection in tumor thorough ddPCR, and also that ptDNA can be identified in pre- and postoperative blood samples of patients with early-stage BC.

Furthermore, Chung et al (45) determined ctDNA genomic changes in patients with $\mathrm{ER}^{+}$metastatic $\mathrm{BC}(\mathrm{mBC})$ by hybrid capture-based genomic profiling. They analyzed time-matched ctDNA and tissue samples from 14 patients and
$89 \%$ of mutations in tissues were also present in the in ctDNA, but mutations in ESR1, TP53 and PIK3CA were detected only the in ctDNA samples. The authors therefore considered that this detection of extra mutations in ctDNA could support liquid biopsy use in detecting heterogenous metastatic sites in $\mathrm{ER}^{+} \mathrm{mBC}$. Stover et al (46), conducted a retrospective study using cfDNA to determine somatic copy-number variations in primary and metastatic tumors and cfDNA tumor fractions in patients with triple-negative $\mathrm{BC}$. Their work highlighted that cfDNA tumor fractions $\geq 10 \%$ were significantly associated with worse survival.

In conclusion, the results of the present study add to the mounting evidence supporting the utility of ctDNA as complementary analysis to tissue biopsy for thorough molecular profiling of tumor variants. Finally, it was demonstrated that next-generation ctDNA sequencing is useful for monitoring the changes in somatic variants with pathogenic effects in patients with $\mathrm{BC}$, and this analysis can supplement the results of tumor tissue biopsy.

\section{Acknowledgements}

Not applicable.

\section{Funding}

The present study was supported by the Slovak Research and Development Agency (grant nos. 14-0815 and 16-0021), the Slovak Scientific Grant Agency (grant no. 1/0124/17) and the Grant of Comenius University (grant nos. 13/2019 and 35/2019).

\section{Availability of data and materials}

All data generated or analyzed during the present study are available from the corresponding author on reasonable request.

\section{Authors' contributions}

$\mathrm{KZ}$, MJ and KD formulated and designed the study. ZL and $M B$ collected the samples, obtained patients' written informed consents, and created patients' database. $\mathrm{KZ}$ and MJ analyzed the samples and carried out NGS analysis. MG recommended bioinformatic tools for NGS data analysis and provided bioinformatic support. $\mathrm{KZ}$ and $\mathrm{MJ}$ interpreted the data and wrote the manuscript. KD and ZD acquired funding, supervised the analyses and revised the manuscript.

\section{Ethics approval and consent to participate}

The authors followed all required ethical guidelines and received approval from the Ethics Committee of Jessenius Faculty of Medicine, Comenius University (IORG0004721) under project identification code EC 1525/2014. Written informed consent was obtained from all patients and the study was conducted in accordance with the Declaration of Helsinki.

\section{Patient consent for publication}

The authors gained informed consent for publication of the dataset from participants at the point of recruitment to the 
study and publication of these data does not compromise anonymity or confidentiality or breach local data protection laws, for the dataset to be considered for publication.

\section{Competing interests}

The authors declare that they have no competing interests.

\section{References}

1. Bray F, Ferlay J, Soerjomataram I, Siegel RL, Torre LA and Jemal A: Global cancer statistics 2018: GLOBOCAN estimates of incidence and mortality worldwide for 36 cancers in 185 countries. CA Cancer J Clin 68: 394-424, 2018.

2. Ellsworth RE, Blackburn HL, Shriver CD, Soon-Shiong P and Ellsworth DL: Molecular heterogeneity in breast cancer: State of the science and implications for patient care. Semin Cell Dev Biol 64: 65-72, 2017

3. Liang DH, Ensor JE, Liu Z, Patel A, Patel TA, Chang JC and Rodriguez AA: Cell-free DNA as a molecular tool for monitoring disease progression and response to therapy in breast cancer patients. Breast Cancer Res Treat 155: 139-149, 2016.

4. Wang R, Li X, Zhang H, Wang K and He J: Cell-free circulating tumor DNA analysis for breast cancer and its clinical utilization as a biomarker. Oncotarget 8: 75742-75755, 2017.

5. Lo YM, Zhang J, Leung TN, Lau TK, Chang AM and Hjelm NM: Rapid clearance of fetal DNA from maternal plasma. Am J Human Genet 64: 218-224, 1999.

6. Chang Y, Tolani B, Nie X, Zhi X, Hu M and He B: Review of the clinical applications and technological advances of circulating tumor DNA in cancer monitoring. Ther Clin Risk Manag 13: 1363-1374, 2017.

7. Wan JCM, Massie C, Garcia-Corbacho J, Mouliere F, Brenton JD, Caldas C, Pacey S, Baird R and Rosenfeld N: Liquid biopsies come of age: Towards implementation of circulating tumour DNA. Nat Rev Cancer 17: 223-238, 2017.

8. Bronkhorst AJ, Ungerer V and Holdenrieder S: The emerging role of cell-free DNA as a molecular marker for cancer management. Biomol Detect Quantif 17: 100087, 2019.

9. Snyder MW, Kircher M, Hill AJ, Daza RM and Shendure J: Cell-free DNA comprises an in vivo nucleosome footprint that informs its Tissues-Of-Origin. Cell 164: 57-68, 2016.

10. Moss J, Magenheim J, Neiman D, Zemmour H, Loyfer N, Korach A, Samet Y, Maoz M, Druid H, Arner P, et al: Comprehensive human cell-type methylation atlas reveals origins of circulating cell-free DNA in health and disease. Nat Commun 9: 5068, 2018

11. Merker JD, Oxnard GR, Compton C, Diehn M, Hurley P,Lazar AJ, Lindeman N, Lockwood CM, Rai AJ, Schilsky RL, et al: Circulating tumor DNA analysis in patients with cancer: American Society of Clinical Oncology and College of American Pathologists Joint Review. J Clin Oncol 36: 1631-1641, 2018.

12. Dawson SJ, Tsui DW, Murtaza M, Biggs H, Rueda OM, Chin SF, Dunning MJ, Gale D, Forshew T, Mahler-Araujo B, et al Analysis of circulating tumor DNA to monitor metastatic breast cancer. N Engl J Med 368: 1199-1209, 2013.

13. Eigeliene N, Saarenheimo J and Jekunen A: Potential of liquid biopsies for breast cancer screening, diagnosis, and response to treatment. Oncology 96: 115-124, 2019.

14. Buono G, Gerratana L, Bulfoni M, Provinciali N, Basile D, Giuliano M, Corvaja C, Arpino G, Del Mastro L,De Placido S, et al: Circulating tumor DNA analysis in breast cancer: Is it ready for prime-time? Cancer Treat Rev 73: 73-83, 2019.

15. Xu C, Gu X, Padmanabhan R, Wu Z, Peng Q, DiCarlo J and Wang Y: smCounter2: An accurate low-frequency variant caller for targeted sequencing data with unique molecular identifiers. Bioinformatics 35: 1299-1309, 2019.

16. Beaver JA, Jelovac D, Balukrishna S, Cochran RL, Croessmann S, Zabransky DJ, Wong HY, Valda Toro P, Cidado J, Blair BG, et al: Detection of cancer DNA in plasma of patients with Early-stage breast cancer. Clin Cancer Res 20: 2643-2650, 2014.

17. Chae YK, Davis AA, Jain S, Santa-Maria C, Flaum L, Beaubier N, Platanias LC, Gradishar W, Giles FJ and Cristofanilli M: Concordance of genomic alterations by next-generation sequencing in tumor tissue versus circulating tumor DNA in breast cancer. Mol Cancer Ther 16: 1412-1420, 2017.
18. Page K, Guttery DS, Fernandez-Garcia D, Hills A, Hastings RK, Luo J, Goddard K, Shahin V, Woodley-Barker L, Rosales BM, et al: Next generation sequencing of circulating Cell-free DNA for evaluating mutations and gene amplification in metastatic breast cancer. Clin Chem 63: 532-541, 2017.

19. Shatsky R, Parker BA, Bui NQ, Helsten T, Schwab RB, Boles SG and Kurzrock R: Next-generation sequencing of tissue and circulating tumor DNA: The UC San Diego Moores center for personalized cancer therapy experience with breast malignancies. Mol Cancer Ther 18: 1001-1011, 2019.

20. Cheng FT, Lapke N, Wu CC, Lu YJ, Chen SJ, Yu PN, Liu YT and Tan KT: Liquid biopsy detects relapse five months earlier than regular clinical follow-up and guides targeted treatment in breast cancer. Case Rep Oncol Med 2019: 6545298, 2019

21. Coombes RC, Page K, Salari R, Hastings RK, Armstrong A, Ahmed S, Ali S, Cleator S, Kenny L, Stebbing J, et al: Personalized detection of circulating tumor DNA antedates breast cancer metastatic recurrence. Clin Cancer Res 25: 4255-4263, 2019.

22. Garcia-Murillas I, Chopra N, Comino-Méndez I, Beaney M, Tovey H, Cutts RJ, Swift C, Kriplani D, Afentakis M, Hrebien S, et al: Assessment of molecular relapse detection in Early-stage breast cancer. JAMA Oncol 5: 1473, 2019.

23. Ma F, Zhu W, Guan Y, Yang L, Xia X, Chen S, Li Q, Guan X, Yi Z, Qian H, et al: ctDNA dynamics: A novel indicator to track resistance in metastatic breast cancer treated with anti-HER2 therapy. Oncotarget 7: 66020-66031, 2016.

24. Zerbino DR, Achuthan P, Akanni W, Amode MR, Barrell D, Bhai J, Billis K, Cummins C, Gall A, Girón CG, et al: Ensembl 2018. Nucleic Acids Res 46: D754-D761, 2018

25. Kopanos C, Tsiolkas V, Kouris A, Chapple CE, Aguilera MA, Meyer R and Massouras A: VarSome: The human genomic variant search engine. Bioinformatics 35: 1978-1980, 2019.

26. Richards S, Aziz N, Bale S, Bick D, Das S, Gastier-Foster J, Grody WW, Hegde M, Lyon E, Spector E, et al: Standards and guidelines for the interpretation of sequence variants: A joint consensus recommendation of the American College of Medical Genetics and Genomics and the Association for Molecular Pathology. Genet Med 17: 405-423, 2015.

27. Tate JG, Bamford S, Jubb HC, Sondka Z, Beare DM, Bindal N, Boutselakis H, Cole CG, Creatore C, Dawson E, et al: COSMIC: The catalogue of somatic mutations in cancer. Nucleic Acids Res 47: D941-D947, 2019.

28. Ades F, Zardavas D, Bozovic-Spasojevic I, Pugliano L, Fumagalli D, de Azambuja E, Viale G, Sotiriou C and Piccart M: Luminal B breast cancer: Molecular characterization, clinical management, and future perspectives. J Clin Oncol 32: 2794-2803, 2014.

29. Fallahpour S, Navaneelan T, De P and Borgo A: Breast cancer survival by molecular subtype: A population-based analysis of cancer registry data. CMAJ Open 5: E734-E739, 2017.

30. Russnes HG, Lingjærde OC, Børresen-Dale AL and Caldas C: Breast cancer molecular stratification. Am J Pathol 187: 2152-2162, 2017.

31. Halperin RF, Liang WS, Kulkarni S, Tassone EE, Adkins J, Enriquez D, Tran NL, Hank NC, Newell J, Kodira C, et al: Leveraging spatial variation in tumor purity for improved somatic variant calling of archival tumor only samples. Front Oncol 9: 119, 2019.

32. Lee LA, Arvai KJ and Jones D: Annotation of sequence variants in cancer samples. J Mol Diagn 17: 339-351, 2015.

33. The Cancer Genome Atlas Network: Comprehensive molecular portraits of human breast tumours. Nature 490: 61-70, 2012.

34. Zhang HY, Liang F, Jia ZL, Song ST and Jiang ZF: PTEN mutation, methylation and expression in breast cancer patients. Oncol Lett 6: 161-168, 2013.

35. Carbognin L, Miglietta F, Paris I and Dieci MV: Prognostic and predictive implications of PTEN in breast cancer: Unfulfilled promises but intriguing perspectives. Cancers 11: 1401, 2019.

36. Olivier M, Langerød A, Carrieri P, Bergh J, Klaar S, Eyfjord J, Theillet C, Rodriguez C, Lidereau R, Bièche I, et al: The clinical value of somatic TP53 gene mutations in 1,794 patients with breast cancer. Clin Cancer Res 12: 1157-1167, 2006.

37. Desmedt C, Voet T, Sotiriou C and Campbell PJ: Next-generation sequencing in breast cancer: First take home messages. Curr Opin Oncol 24: 597-604, 2012.

38. Bertheau P, Lehmann-Che J, Varna M, Dumay A, Poirot B, Porcher R, Turpin E, Plassa LF, de Roquancourt A, Bourstyn E, et al: p53 in breast cancer subtypes and new insights into response to chemotherapy. Breast 22 (Suppl 2): S27-S29, 2013. 
39. Kodahl AR, Ehmsen S, Pallisgaard N, Jylling AMB, Jensen JD, Laenkholm AV, Knoop AS and Ditzel HJ: Correlation between circulating cell-free PIK3CA tumor DNA levels and treatment response in patients with PIK3CA-mutated metastatic breast cancer. Mol Oncol 12: 925-935, 2018.

40. Zardavas D, Te Marvelde L, Milne RL, Fumagalli D, Fountzilas G, Kotoula V, Razis E, Papaxoinis G, Joensuu H, Moynahan ME, et al: Tumor PIK3CA genotype and prognosis in early-stage breast cancer: A pooled analysis of individual patient data. J Clin Oncol 36: 981-990, 2018.

41. Alborelli I, Generali D, Jermann P, Cappelletti MR, Ferrero G, Scaggiante B, Bortul M, Zanconati F, Nicolet S, Haegele J, et al: Cell-free DNA analysis in healthy individuals by next-generation sequencing: A proof of concept and technical validation study. Cell Death Dis 10: 534, 2019

42. De Mattos-Arruda L, Weigelt B, Cortes J, Won HH, Ng CKY, Nuciforo P, Bidard FC, Aura C, Saura C, Peg V, et al: Capturing intra-tumor genetic heterogeneity by de novo mutation profiling of circulating cell-free tumor DNA: A proof-of-principle. Ann Oncol 25: 1729-1735, 2014.

43. Finzel A, Sadik H, Ghitti G and Laes JF: The combined analysis of solid and liquid biopsies provides additional clinical information to improve patient care. JCMT 4: 21, 2018.
44. Rodriguez BJ, Córdoba GD, Aranda AG, Álvarez M, Vicioso L Pérez CL, Hernando C, Bermejo B, Parreño AJ, Lluch A, et al: Detection of TP53 and PIK3CA mutations in circulating tumor DNA using next-generation sequencing in the screening process for early breast cancer diagnosis. J Clin Med 8: 1183, 2019.

45. Chung JH, Pavlick D, Hartmaier R, Schrock AB, Young L, Forcier B, Ye P, Levin MK, Goldberg M, Burris H, et al: Hybrid capture-based genomic profiling of circulating tumor DNA from patients with estrogen receptor-positive metastatic breast cancer. Ann Oncol 28: 2866-2873, 2017.

46. Stover DG, Parsons HA, Ha G, Freeman SS, Barry WT, Guo H, Choudhury AD, Gydush G, Reed SC, Rhoades J, et al: Association of cell-free DNA tumor fraction and somatic copy number alterations with survival in metastatic Triple-Negative breast cancer. J Clin Oncol 36: 543-553, 2018.

This work is licensed under a Creative Commons Attribution-NonCommercial-NoDerivatives 4.0 International (CC BY-NC-ND 4.0) License. 